\title{
EFFECTS OF SOME COMMON CATIONS ON ELECTRORETINOGRAM OF THE TOAD
}

\author{
TARO FURUKAWA AND ISAO HANAWA* \\ Department of Physiology, Osaka City University Medical School, Abeno-ku, Osaka, Japan
}

Effects of $\mathrm{K}^{+}$on the $b$-wave of ERG have long been well known, but little has been known of other cations, especially of $\mathrm{Na}^{+}$. An important role played by $\mathrm{Na}^{+}$in the generation of action potential in nerve and muscle was thoroughly established by Hodgkin et al. (4). Recently, Svaetichin reported in his paper (5) that Ringer's fluid had an unfavorable effect on frog ERG, and he used isotonic glucose solution instead of Ringer's as a bathing fluid of the frog retina. Judging from this, $\mathrm{Na}^{+}$would be quite dispensable for the generation of ERG. In view of these somewhat contradictory results, a systematic study seems desirable on the action of some common cations on ERG, although the mode of generation of ERG is considered to be different in various points from that of action potentials in nerve and muscle. For this purpose, the present research has been performed with isolated retinas of the toad. Since the preparation was thin, its ionic milieu could be changed promptly and the effects of the absence of an ion, as well as those of presence could be easily detected.

\section{METHOD}

Eyes of the Japanese toad (Bufo vulgaris formosus) were used. Animals were dark adapted before the experiments.

Preparation of the isolated retina. In preparing the isolated retina, descriptions of Svaetichin (5) were mostly followed. An eye-ball was excised and placed with its corneal side downwards on a small rubber block. A small cut was made to the sclera in the equatorial region through which the blade of a scissors for iridectomy was introduced, and the sclera was removed in small pieces. The eye-ball was then placed on a sheet of filter paper moistened with Ringer's, with its corneal side upwards, and cut in two at the equatorial region. The upper half of the eye-ball was removed, leaving the retina with attached chorioidea, which became flattened on the filter paper by its own weight. Finally, a surplus part of the filter paper around the retina was cut away, so that the rest part of it served as a holder for the retina. All the manipulations above described were carried out under a white dim light.

Preparation box. The preparation box was made of metaacryl resin plates and composed of two chambers, which were connected by a small glass tube at

Received for publication July 31, 1955.

* 古河太郎, 塙 功 
the bottom (fig. 1). The isolated retina placed on a small piece of filter paper was laid half immersed in Ringer's at the bottom of one of these chambers, and a silver-silver chloride electrode was placed in the other. Since the connecting glass tube was already filled with Ringer's, this electrode served as a lead electrode from the outer surface of the isolated retina. The other electrode, also made of silver-silver chloride, was attached to the cover of the chamber and it was in contact with the upper surface (vitreous side) of the retina by a short moistened cotton thread. A stimulating light was thrown on the vitreous side of the retina through the cover of the chamber.

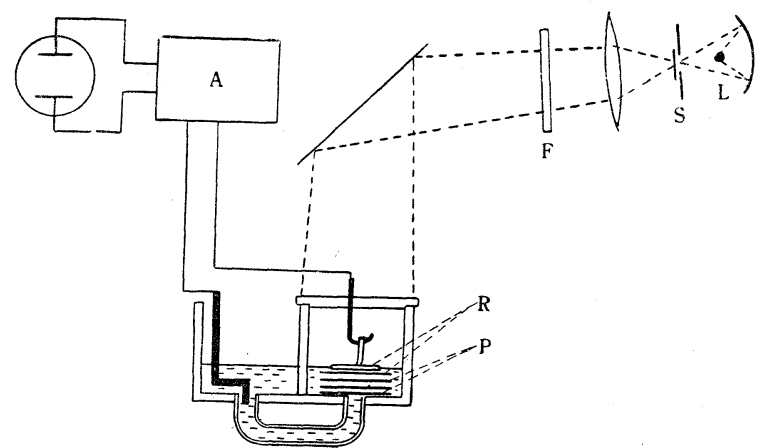

FIG. 1. Experimental arrangements.

$A$ : C-R coupled amplifier. $F$ : Neutral filter. $L$ : Light source. $P$ : Sheets of filter paper which served to maintain the retina at the fluid surface. $R$ : Isolated retina with its holder (one sheet of filter paper). $S$ : Electromagnetic shutter.

Exchange of the bathing fluid and application of drugs. The fluid bathing the retina was exchanged in order to study the effects of ionic milieu on ERG. For this purpose, the retina was removed together with its holder (filter paper) to another box and placed on a sheet of dry filter paper, which absorbed fluid attached to the retina and its holder. The retina was returned to the preparation box, after the Ringer's in the preparation box was thoroughly washed by a test solution.

When the retina was totally immersed in the test solution, the effect of it appeared rather rapidly but it was often the case that retina soon deteriorated, probably due to an oxygen lack; so the test solution had to be saturated beforehand with oxygen and the time of immersion should have been limited. There was another drawback in this method, that the retina was often torn off from the chorioidea and folded up in itself, and once this happened its ERG was almost abolished. These shortcomings could be avoided by placing the retina on the surface of the fluid supported by several sheets of filter papers (fig. 1). Since the retina, in this case, was only half immersed in the solution, the effects of the test solution appeared relatively slowly, but the danger of anoxia and abrasion of the retina was totally avoided. Also the actions of drugs were investigated by a similar method. 
Stimulating light. The stimulating light was of white colour. Its original intensity was about 500 Lux, which was reduced by neutral filters inserted in the pathway of light. Usually light of 1-2 Lux was used. Illumination was switched on and off by an electromagnetic shutter, which was controlled by an electronic device. The onset of illumination was synchronized with the horizontal sweep of the c.r.o. and the duration of illumination could be varied from $1 / 10$ to 2 sec. However, short flashes of light were usually used, because of their relatively slight effects on the state of adaptation. A C-R coupled amplifier was used, the time constant of which was $1 \mathrm{sec}$. Also a D-C coupled amplifier was used in later experiments.

RESULTS

\section{A. General Remarks}

A typical example of ERG recorded in the present study is shown in fig. 2. Here, as the retina was dark adapted and the stimulating light was weak and of short duration, off-effect was not seen. $C$-wave was more or less present, but this was out of scope of this study, for the amplifier was C-R coupled. The height of $b$ wave was generally $0.3-0.5 \mathrm{mV}$ in good preparations, but sometimes a little smaller values were encountered. The condition of the retina remained usually unchanged for several hours. According to Svaetichin (5), Ringer's was said to have an unfavorable effect on the retina. On the contrary, it was proved

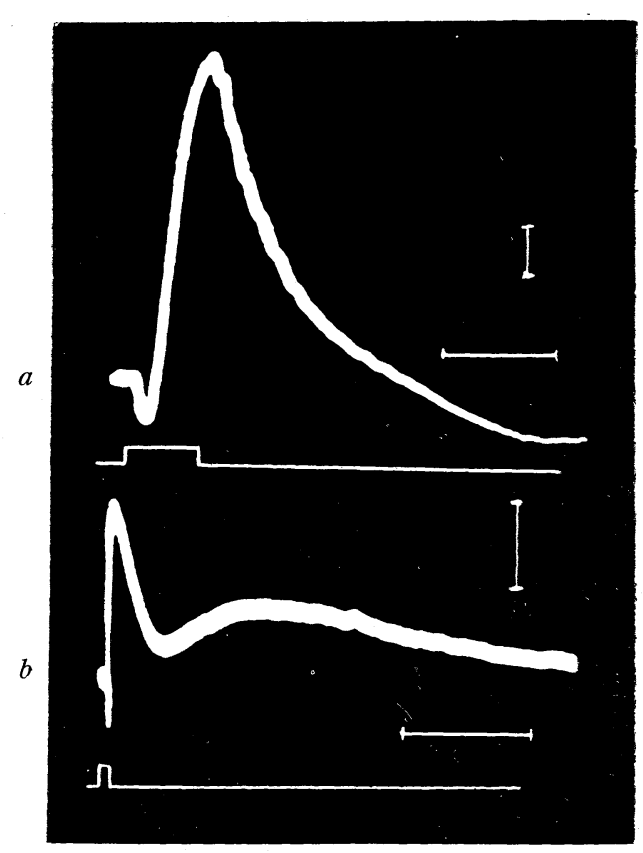
in our experiments that Ringer's was a good substitution fluid for the retinal tissue. The retina half immersed in this solution responded to light quite normally for many hours.

FIG. 2. ERG of the isolated retina. Off-effect is not seen under the condition here employed (see text). Duration of illumination is marked under each record. Voltage calibration: 0.1 $\mathrm{mV}$.

(a): C-R coupled amplification. Time: $0.5 \mathrm{sec}$ (b): D-C coupled amplification. Slow time base. Time: 5 sec. 
"Retinal shock". When ERG was examined shortly after the preparation was made, a mostly negative deflection was often observed. Presumably this phenomenon is due to mechanical disturbances exerted on the retina in the

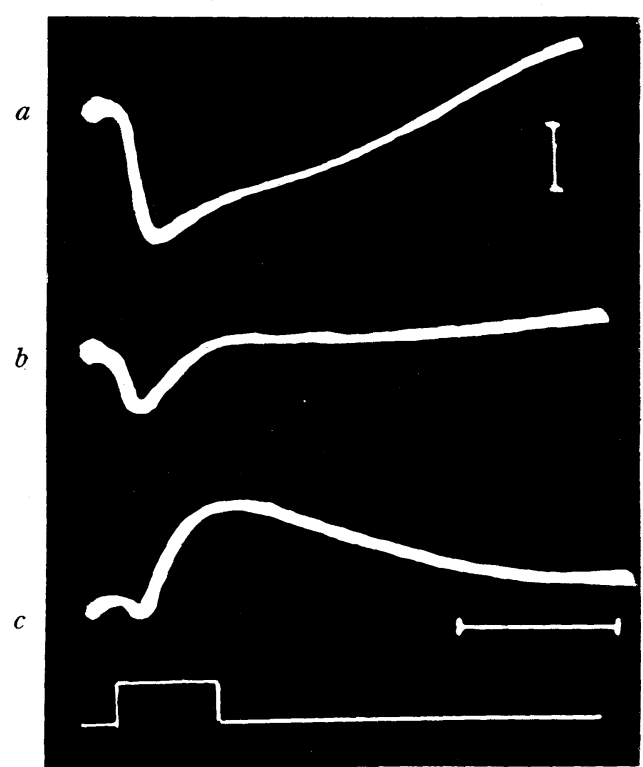
course of isolation, but it was of a temporary nature in almost all cases, and the ERG became normal in 15-30 minutes when the retina was preserved half immersed in Ringer's. We call this phenomenon "retinal shock" from its apparent similarity to the shock of the spinal cord. An example is shown in fig. 3.

FIG. 3. "Retinal shock."

(a): ERG recorded shortly after the preparation of the isolated retina. Almost negative deflection. $(b): 5 \mathrm{~min}$. after $(a) . \quad(c)$ : $35 \mathrm{~min}$. $\operatorname{after}(a)$. Almost normal response. Voltage calibration : $0.1 \mathrm{mV}$. Time : $0.5 \mathrm{sec}$.

Reversibility of drug action. In the experiments on the isolated retina, actions of various drugs were generally reversible so long as their concentrations were suitably selected. In certain casses the action of drug disappeared very rapidly in 1 to 2 minutes after the retina was returned to Ringer's.

\section{B. Relation between ERG and Ions}

\section{ERG in glucose Ringer}

The isolated retina was immersed in glucose Ringer and the changes in ERG were investigated from time to time by test flashes. The constitution of glucose Ringer is shown in table $1(b)$. Fig. 4 shows a typical course of the experiment. The first sign after the application of glucose Ringer was a decrease in the height of $b$-wave and a relative increase of $a$-wave in size. Generally $b$-wave became smaller and smaller, till it completely disappeared (fig. $4(c)$ ). Here, there remained only a negative deflection which seemed to correspond to $P_{I I I}$ in Granit's designation. The time needed for a complete abolition of $b$-wave varied from 20 to 80 minutes.

When the retina was returned to normal Ringer, $b$-wave reappeared in about 10 minutes, and ERG was restored gradually its normal form. But the amplitude of the ERG was somewhat smaller than that obtained at the beginning of the experiment. This was shown in fig. $4(d)$ and $(e)$.

These findings seemed to indicate at first sight that, glucose, like $\mathrm{KCl}$, would have had a depressing effect on $b$-wave. But this assumption has been denied by the following experiments. 


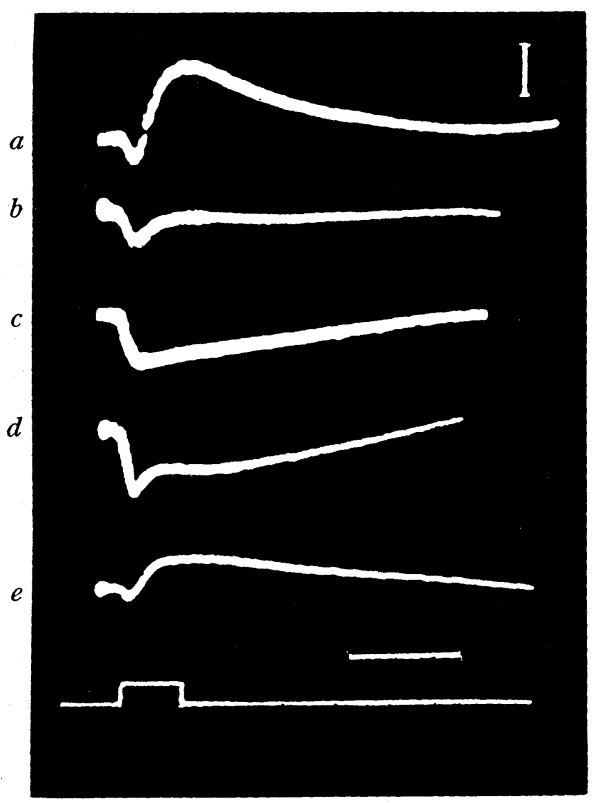

FIG. 4. Effects of Na-free medium (glucose Ringer) on ERG.

(a): 1 min. after the application of glucose Ringer. Almost normal response. (b): After $20 \mathrm{~min}$. (c): After $40 \mathrm{~min}$; $60 \mathrm{~min}$. after the application of glucose Ringer, the retina was returned to normal Ringer. $(d)$ : $10 \mathrm{~min} .,(e): 60 \mathrm{~min}$. after the application of Ringer's. Voltage calibration: $0.1 \mathrm{mV}$. Time: $0.5 \mathrm{sec}$.

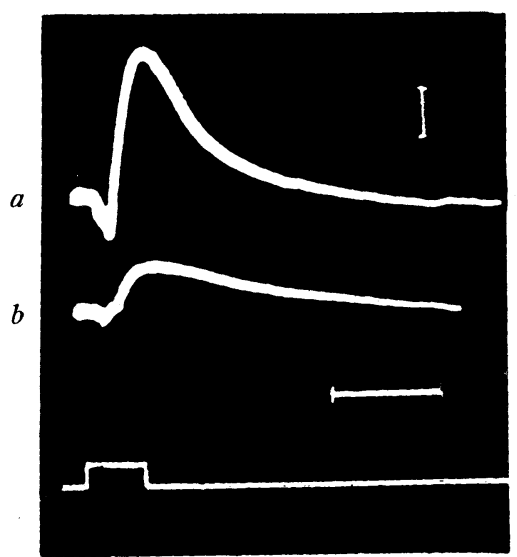

FIG. 5. Effects of glucose on ERG.

$3.5 \%$ glucose was dissolved in Ringer's. This hypertonic solution was applied to the retina.

$(a): 2$ min., $(b): 30$ min. after the application. Voltage calibration : 0.1 $\mathrm{mV}$. Time : $0.5 \mathrm{sec}$.

(a) Instead of glucose Ringer a hypertonic solution was employed, in which $3.5 \%$ glucose was dissolved in normal Ringer. When this solution was applied to the retina, ERG changed its amplitude but never its time course. As is shown in fig. 5, the amplitude of ERG decreased with the lapse of time after application of the hypertonic solution. Similar results were obtained with the mixture of normal Ringer and glucose Ringer in an equal amount.

(b) The retina was first immersed in glucose Ringer, and after $b$-wave had disappeared, either of the above solutions was applied to it. Fig. $6(b)$ shows the negative component of ERG isolated in glucose Ringer. When the mixture of normal Ringer and glucose Ringer was applied, $b$-wave reappeared as shown in $(c)$ and $(d)$.

From these findings it became clear that the absence of $\mathrm{Na}^{+}$rather than the action of glucose played a principal role in the abolition of $b$-wave in glucose Ringer. But a slight possibility is that glucose itself may have a weak depressing action on $b$-wave. 
The isolated negative component in glucose Ringer, however, became gradually smaller with the lapse of time. That $\mathrm{Na}^{+}$is also necessary for the generation of negative component of ERG, will be shown in a later section.

FIG. 6. (a): Normal ERG, the retina was immersed in Ringer's. $(b)$ : 85 min. after the application of glucose Ringer. Directly after this was taken, a mixed solution of Ringer's and glucose Ringer ( $1: 1)$ was applied to the retina. (c): 15 min., $(d): 60$ min. after the application of the mixed solution. Voltage calibration: $0.1 \mathrm{mV}$. Time : $0.5 \mathrm{sec}$.

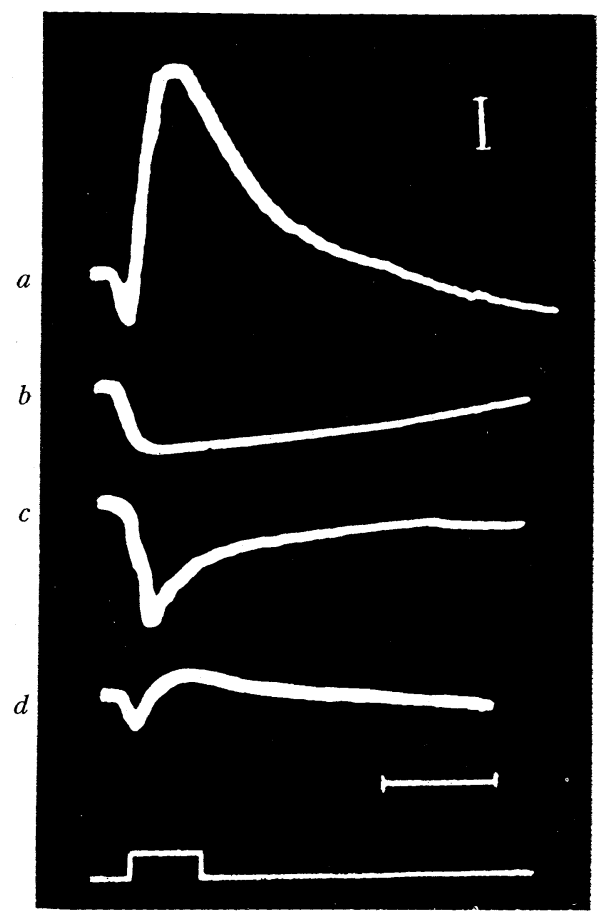

\section{Effects of other Na free media}

Since the effects of glucose Ringer were proved to be attributable to the absence of $\mathrm{Na}^{+}$, tests were made on other Na-free media which were often used in electrophysiology experiments. These were isotonic sucrose $(7 \%)$, choline chloride, and hexamethonium bromide solutions, to which suitable amounts of $\mathrm{Ca}^{++}$and $\mathrm{K}^{+}$were added. The retina was immersed in one of these solutions, as in the case of glucose Ringer, and ERG was recorded periodically. But the results were not quite in accordance with our expectation, and both $a$ - and $b$ wave became smaller and smaller till they finally disappeared. On the whole this was common to the above three solutions, making them unsuitable for the isolation of the negative component. But in the case of sucrose, $b$-wave disappeared a little earlier than $a$-wave so that the result was somewhat similar to that of glucose.

Also it was proved that these substances did not have any power of regenerating $b$-wave when applied to the retina, after its $b$-wave had abolished in glucose Ringer.

\section{Effects of ions on an isolated negative component}

In order to clarify whether the absence of ions would be the cause of a gradual diminution in the negative component in glucose Ringer, effects of some kinds of ions were tested, when added to the glucose Ringer. First, $\mathrm{K}^{+}$was added to the solution. After $b$-wave had disappeared in glucose Ringer, bathing 
fluid wás replaced by glucose Ringer containing $40 \mathrm{~mm} \mathrm{~K}^{+}$. Here, in obtaining isotonicity, a proper amount of glucose was removed. But the results were the same as when no excess $\mathrm{K}^{+}$was added to the solution, and the electrical response became smaller in about 50 minutes. Similar results were obtained in 60-80 $\mathrm{mm} \mathrm{K}^{+}$.

Next, effects of $\mathrm{Ca}^{++}$were tested. $40 \mathrm{~mm} \mathrm{Ca}^{++}$were added to glucose Ringer, but this did not improve the situation any more.

Now, $\mathrm{Na}^{+}$were added to the solution. In the experiment shown in fig. 6 , glucose Ringer containing $55 \mathrm{~mm} \mathrm{Na}^{+}$was employed. It was to be noted that the first sign of change was an increase in the amplitude of negative deflection. But this augmentative effect on the negative component became obscure, because of a rapid reappearance of $b$-wave. So it was necessary to adopt some measure to suppress the reappearance of $b$-wave. For this purpose, a suitable amount (20 $\mathrm{mm}$ ) of sodium asparate was added to the solution. This substance was found by one of the present authors (I.H.) to have a very strong inhibitory effect on $b$-wave. It was considered particularly suitable for the present purpose. This action of aspartic acid was presumably based on a metabolic interference of the retina. The composition of the solution which was used for this purpose is shown in table $1(c)$. (This solution will be henceforth called "AGR.") When the glucose Ringer bathing the retina was replaced by "AGR," negative component of ERG became very large. This is shown in fig. 7 . In this, $(b)$ is the isolated negative component obtained by immersing the retina in isotonic glucose

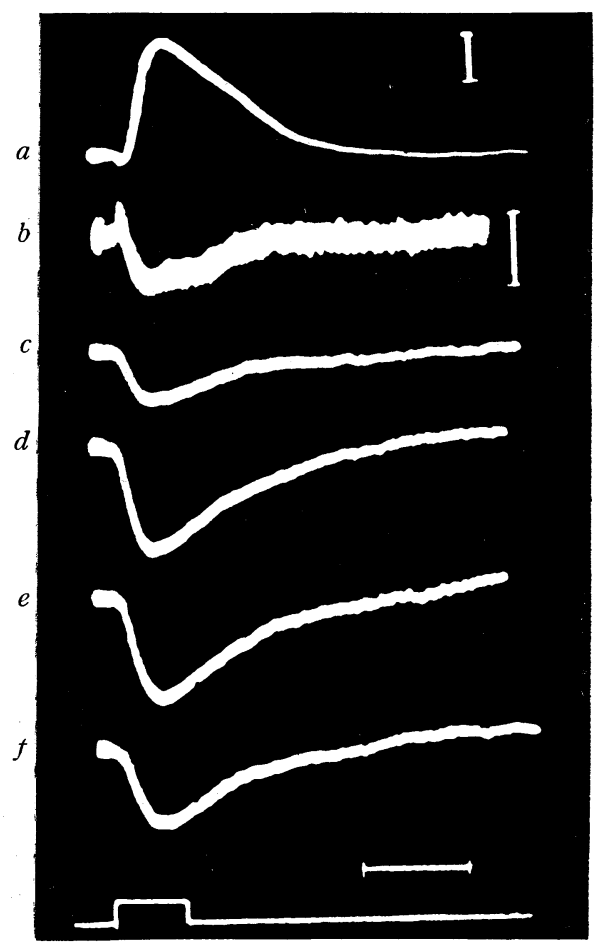

for $30 \mathrm{~min}$. The bathing fluid was changed to "AGR." $(c)$ and $(d)$ are obtained 2 and 10 minutes later. In the latter, the amplitude of the negative component increased clear$l y$, and in $(e)$ and $(f)$ taken 120 and 180 minutes later, no further change was observed in the amplitude. Thus in "AGR" the condition of the retina remained constant for over three hours and the amplitude of ERG, consisting solely of negative component, remained almost unchanged.

FIG. 7. Effects of $\mathrm{Na}^{+}$on negative component of ERG.

(a): Normal ERG, the retina was immersed in Ringer's. $(b)$ : $30 \mathrm{~min}$. after the application of glucose Ringer to the retina. Directly after this was taken, "AGR" was applied to the retina. $(c)$ : 2 min., $(d): 10$ min., $(e): 120$ min., $(f)$ : $180 \mathrm{~min}$. after the application of “AGR." Voltage calibration: $0.1 \mathrm{mV}$. From $(b)$ to $(f)$, same amplification. Time : 0.5 sec. 
TABLE 1. Composition of the Bathing Fluids

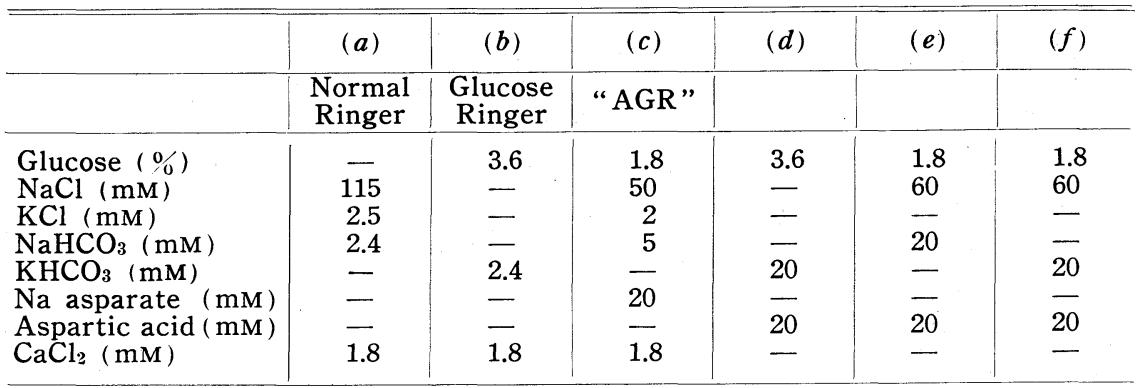

The alternative explanation that aspartic acid per se, and not $\mathrm{Na}^{+}$, might have effects of increasing and maintaining the negative component of ERG, was denied by the following experiments. After negative component was isolated in glucose, bathing fluid was changed to a $20 \mathrm{~mm}$ solution of aspartic acid in isotonic glucose. Since this solution was neutralized $\left(p_{\mathrm{H}}=6\right)$ by adding $20 \mathrm{~mm}$ $\mathrm{KHCO}_{3}$, it contained $20 \mathrm{~mm} \mathrm{~K}^{+}$but no $\mathrm{Na}^{+}$(table $1(d)$ ). The result was that in this solution the isolated negative component became smaller in about 30 to 50 minutes. Next, bathing fluid was exchanged with one which contained a certain amount of $\mathrm{Na}^{+}$in place of glucose. The composition of this solution is shown in table $1(e)$ or $(f)$. Then, about 5 to 10 minutes later, negative deflection became larger again. The result was very clear and similar to that shown in fig. 7.

It was concluded from these results that the presence of $\mathrm{Na}^{+}$was essential to the generation of ERG, though the negative component was somewhat more resistant to its absence.

\section{Effects of $K^{+}$and other ions on $E R G$}

(a) Effects of $K^{+}$on ERG

Effects of $\mathrm{KCl}$ on ERG were studied by several authors $(2,7)$. In these studies, however, concentration of $\mathrm{KCl}$ in the retina was not exactly known, nor was reversibility of its action tested. In the experiments performed on the isolated retina, these points were satisfactorily studied.

When the retina was immersed in isotonic solution of $\mathrm{KCl}, \mathrm{ERG}$ soon disappeared, and the recovery of it after the retina was returned to normal Ringer was incomplete.

Next, effects of $60 \mathrm{~mm} \mathrm{~K} \mathrm{~K}^{+}$were studied. The retina was immersed in Ringer's containing $60 \mathrm{~mm} \mathrm{~K} \mathrm{~K}^{+}$(equivalent $\mathrm{Na}^{+}$was removed to maintain isotonicity). In fig. 8, $(a)$ is a record obtained 1 minute after the application of $\mathrm{K}^{+}$, which is similar to that in Ringer's. With the lapse of time, $b$-wave became smaller. (b) and $(c)$ are records observed 15 and 40 minutes, respectively, after the application of $\mathrm{K}^{+}$. In the latter, $b$-wave is almost completely abolished, leaving only a negative deflection, which is rather small in size. After (c) was taken, the retina was returned to Ringer's and about 60 minutes later ERG became quite normal as is shown in $(e) . \quad(d)$ represents an intermediary stage in the course of recovery. 


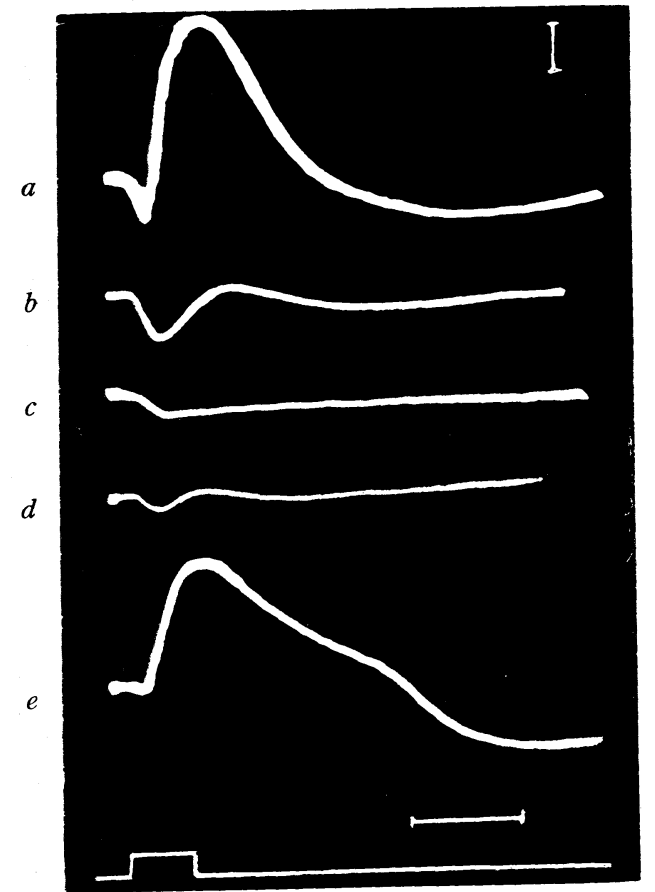

FIG. 8. Reversibility of $K^{+}$action.

A Ringer's containing $60 . \mathrm{mM} K^{+}$was applied to the retina.

$(a)$ : 1 min., $(b): 15$ min., $(c): 40 \mathrm{~min}$. after the application of $\mathrm{K}^{+} .45 \mathrm{~min}$. after the application of $K^{+}$, the retina was returned to Ringer's. $(d): 10$ min., $(e): 120$ min. later. In $(e)$ the duration of illumination was longer (about $1 \mathrm{sec}$.) than indicated below. Voltage calibration : $0.1 \mathrm{mV}$. Time : $0.5 \mathrm{sec}$

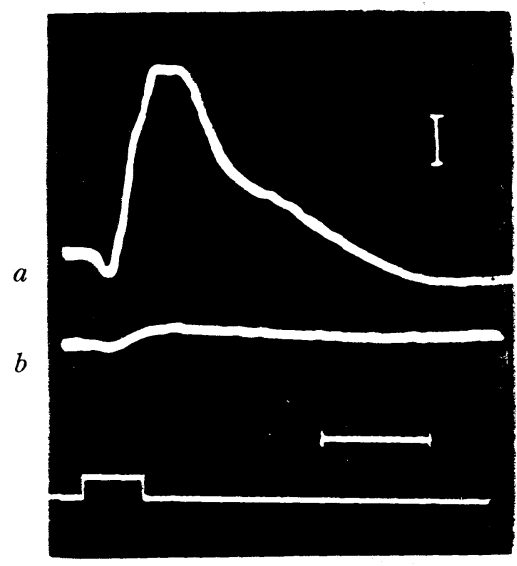

FIG. 9. Effects of $\mathrm{NH}_{4}{ }^{+}$on ERG.

(a): Normal ERG in Ringer's. (b): $40 \mathrm{~min}$. after the application of $20 \mathrm{mM} \mathrm{NH}_{4}$-Ringer. And after the retina was returned to normal Ringer, ERG was not recovered. Voltage calibration: $0.1 \mathrm{mV}$. Time: $0.5 \mathrm{sec}$.

A similar result was obtained also with $40 \mathrm{~mm} \cdot \mathrm{K}^{+}$solution. With $20 \mathrm{~mm}$ $\mathrm{K}^{+}$solution $b$-wave became small, but never disappeared.

(b) Effects of $\mathrm{NH}_{4}^{+}$on $\mathrm{ERG}$

$\mathrm{NH}_{4}{ }^{+}$was found to have a depressing effect both on positive and negative components of ERG. Its effect seemed to be irreversible. Ringer's containing 10-40 mM NH $\mathrm{m}_{4} \mathrm{Cl}$ was applied to the retina. Records $(a)$ and $(b)$ in fig. 9 represent normal ERG in Ringer's and that obtained about 40 minutes after the application of $20 \mathrm{mM} \mathrm{NH}_{4}{ }^{+}$.

\section{(c) Effects of $\mathrm{Li}^{+}$and $\mathrm{Ca}^{++}$on $\mathrm{ERG}$}

$\mathrm{Li}^{+}$was relatively indifferent. It has no specific effect on the retina. But it could not substitute for $\mathrm{Na}^{+}$in the generation of ERG. When the retina was immersed in lithium Ringer ( $\mathrm{Na}$ was replaced by $\mathrm{Li}$ ) ERG became smaller and disappeared completely. 
$\mathrm{Ca}^{++}$seemed to have a weak depressing effect on ERG when applied in concentration of $20-40 \mathrm{~mm}$, but no systematic investigation was so far made concerning the effects of $\mathrm{Ca}^{++}$.

\section{DISCUSSION}

\section{A. Role of $\mathrm{Na}^{+}$in $E R G$}

It was found in this study that $b$-wave of ERG was very susceptible to the lack of $\mathrm{Na}^{+}$in the bathing fluid, and when the retina was immersed in a $\mathrm{Na}$ free medium (isotonic glucose) $b$-wave disappeared first and negative component of ERG remained. But the remained negative component became smaller with the lapse of time. Here, $\mathrm{Na}^{+}$was indispensable in evoking the isolated negative component for a long period of time.

Thus, $\mathrm{Na}^{+}$was proved to be indispensable for the generation not only of $b$-wave, but of the negative component of ERG, $b$-wave being more susceptible to lack of $\mathrm{Na}^{+}$.

Now, $b$-wave of ERG is known to share certain properties with the action potential in the nerve. For example, both of them suffer depressive effects by the action of $\mathrm{K}^{+}$in certain concentration and by anoxia. Here, susceptibility to Na-lack is added to these properties. But, it is uncertain that the role of $\mathrm{Na}^{+}$in the mechanism of generation of $b$-wave of ERG is also analogous to the action potential of the nerve. In fact, the present study of ERG is not so precise, and the effect of $\mathrm{Na}^{+}$on the amplitude of $b$-wave of ERG is not studied quantitatively. Moreover, there are other properties that are different from the action potential of the nerve, i.e. $b$-wave of ERG is not abolished by the action of narcotics (urethane, alcohol, cocaine, etc.). In the present study, it was found that $\mathrm{Li}^{+}$did not substitute for $\mathrm{Na}^{+}$for the generation of $b$-wave whereas for the generation of action potential of the nerve, $\mathrm{Li}^{+}$was known to substitute for $\mathrm{Na}^{+}$.

Furthermore, action potential of the nerve follows the all or nothing law, but in $b$-wave of ERG it is commonly believed that the stronger the stimulus, the greater the response. From these facts, it seems likely that the mode of generation of $b$-wave of ERG is analogous to the depolarization of the muscleendplate by acetyl-choline. The depolarization of the end-plate by acetyl-choline is a somewhat passive process and does not follow the all or nothing law, and also, presence of $\mathrm{Na}^{+}$in the mediun is said to enhance the reaction (1).

\section{B. Elimination of $b$-Wave}

Many methods have been so far found for the isolation of negative component of ERG and especially Therman's method of dropping $0.5 \% \mathrm{KCl}$ solution in isotonic glucose into the opened eye has been said to give a fairly good result (6). From the view point of the present research, this method seems to have combined the action of Na-lack and excess $\mathrm{K}^{+}$. The effect of Na-lack was discussed in the preceding section. Concerning the action of $\mathrm{K}^{+}$, Granit (2) was of opinion that $\mathrm{K}^{+}$not only suppressed positive component of ERG but somewhat enhanced the negative component. But to the present authors this latter effect seems quite dubious, for as stated in results, $\mathrm{K}^{+}$did not have any power of 
augmenting the negative component isolated by the action of glucose Ringer. In the present research, the stimulating light was a short flash of light, so that the effect of $\mathrm{K}^{+}$on the off-effect was not studied.

Next, it is to be noted that the isolation of negative component in glucose Ringer is complete. And even when various drugs, which were thought to have stimulating effects on $b$-wave, were applied to the retina, no trace of $b$-wave was ever detected so long as there was no $\mathrm{Na}^{+}(3)$. Among those drugs were urethane $(2.5 \%)$, ethyl-alcohol $(5 \%)$, and cocaine $(1 \%)$. On the other hand, since $b$-wave was merely supressed when the negative component was isolated by the action of $\mathrm{K}^{+}$or aspartic acid etc. in the presence of $\mathrm{Na}^{+}, b$-wave reappeared promptly when one of the drugs above mentioned was applied to the retina (3). From these findings, the authors' impression is that in glucose, $b$ wave was not merely suppressed, but the mechanism for its generation was radically inactivated by $\mathrm{Na}$-lack. It was reported that $b$-wave reappeared when $10 \%$ alcohol was applied to the opened eye after its $b$-wave had been eliminated by Therman's method. This is probably due to the fact that in Therman's method $\mathrm{Na}^{+}$was not washed out of the retina, though Na-free solution was dropped into the opened eye. There is relatively abundant blood and lymph in between receptor layer and sclera, and diffusion through the retina is not thought rapid. It is also supposed that exchange of the contents of the opened bulb was not performed satisfactorily. Concerning the action of alcohol, Granit was of opinion that a chief action of alcohol was the suppression of the negative component and a facilitating effect on $b$-wave was of slight degree (2). But, the present authors have a contrary view, i.e. the chief action of alcohol lies in the enhancement of $b$-wave of ERG and the action on the negative component is less, or none. These properties seem to be common to certain narcotics, i.e. alcohol, urethane, and cocaine. The authors are of the opinion that so called isolation of $b$-wave under the action of one of these drugs is only of an apparent phenomenon because following the addition of $\mathrm{K}^{+}$, negative deflection always appears, whereas $\mathrm{K}^{+}$itself has no augmentative effect on the negative component. Since it was proved by the present authors in the study of spectral sensitivity of toad's retina (3), in accordance with Therman (2), that not only the amplitude of $b$-wave, but that of the isolated negative component showed the Purkinje shift, and also since $a$-wave seemed to be evoked primarily by light stimulus, it seems more likely that $b$-wave does not occur independently of $a$-wave.

SUMMARY

Effects of some common cations on electroretinogram (ERG) were studied using the isolated retina of the Japanese toad.

1. The isolated retina could be maintained in good condition for several hours, if preserved half immersed in Ringer's. Ringer's was a favorable substituting fluid for the retina.

2. The presence of $\mathrm{Na}^{+}$was indispensable for both $a$ - and $b$-wave of ERG. But $b$-wave was far more susceptible to the lack of $\mathrm{Na}^{+}$in the medium. When the retina was immersed in an isotonic glucose solution, $b$-wave disappeared and only the negative component remained. 
3. The amplitude of this isolated negative component was found to increase if $\mathrm{Na}^{+}$was added to the bathing fluid. This was shown very clearly if reappearance of $b$-wave was avoided by adding a suitable amount of aspartic acid to the bathing fluid. Aspartic acid was found to have a very strong and specific depressant action on $b$-wave of ERG.

4. $\mathrm{K}^{+}$had a depressing effect on $b$-wave of ERG, but it seemed to have no augmentative effect on negative component.

5. Methods of eliminating $b$-wave from ERG and some properties of the thus isolated negative component were discussed.

The authors' sincere thanks are due to Prof. Y. Hosoya and Prof. T. Otani (Kyoto University) for their kind suggestions in finishing this paper.

\section{REFERENCES}

1. FAtt, P. J. Physiol. 111: 408, 1950.

2. Granit, R. Sensory Mechanisms of the Retina. Oxford: Oxford University Press, 1947.

3. HANA.WA, I. AND T. FURUkAWA. Unpublished data.

4. Hodgkin, A. L. Biol. Rev. 26 : 339, 1951.

5. Svaetichin, G. Acta Physiol. Scand. 29, Supp. 106: 538, 1953.

6. Therman, P. O. Acta Soc. Sci. Fenn. N.S.B. II : No. 1, 1938.

7. TOIDA, N., K. OHKi AND H. A. KURIYAma. Kyusyu Daigaku Seisan Kenkyuzyo Hokoku (Bulletin of the Research Institute of Science and Industry) No. 13, 47, 1954 (Japanese). 